\title{
Identification of Benzoylcarnitine in the Urine of a Patient of Hyperammonemia
}

\author{
Toru Sakuma, Kiyohumi Asai, Takashi Ichiki, Naruji \\ Sugiyama, Kiyoshi Kidouchi* and Yoshiro Wada \\ Department of Pediatrics, Nagoya City University Medical \\ School, Nagoya 46\%, and *Department of Pediatrics, \\ Higashi City General Hospital, Nagoya 464
}

Sakuma, T., Asai, K., Ichiki, T., Sugiyama, N., Kidouchi, K. and Wada, Y. Identification of Benzoylcarnitine in the Urine of a Patient of Hyperammonemia. Tohoku J. Exp. Med., 1989, 159 (2), 147-151 — Benzoylcarnitine was identified in the urine of a patient with a carbamoyl-phosphate synthase I deficiency for whom sodium benzoate and L-carnitine had been used to treat hyperammonemia. This is a newly identified metabolite of benzoate. Its excretion in the urine was increased day by day at the administration of both sodium benzoate and Lcarnitine from 0.10 to $2.25 \mathrm{mmol} / \mathrm{g}$ creatinine. Since there is the possibility of a secondary carnitine deficiency and an increase of benzoyl toxicity after long-term therapy with benzoate supplementation and protein restriction, it is important to monitor the urinary excretion of benzoylcarnitine._- benzoylcarnitine; deuterium labeled carnitine; sodium benzoate; hyperammonemia

Ammonia is excreted in the form of hippurate via glycine conjugation with benzoyl-CoA, so it has been preferred to treat patients of hyperammonemia with sodium benzoate. But it was reported that all patients with partial ornithine transcarbamylase deficiency had secondary carnitine deficiency, and L-carnitine supplement was effective to reduce the frequency of hyperammonemic attacks (Matsuda et al. 1987). As it is speculated that carnitine conjugated with benzoyl-CoA may be also excreted in urine, and it may be one cause of hypocarnitinemia, we analized acylcarnitine in the urine of a patient treated with sodium benzoate.

\section{Materials and Methods}

\section{Materials}

Urine samples of a patient with carbamoyl-phosphate synthase I deficiency, who had been treated intravenously with sodium benzoate $(250 \mathrm{mg} / \mathrm{kg} /$ day $)$ and L-carnitine $(100 \mathrm{mg} /$ $\mathrm{kg}$ /day), were collected and stored at $-20^{\circ} \mathrm{C}$ until analysis.

All the reagents used were of reagent grade. Labeled carnitine (D3-Mel-N : carnitine)

Received September 1, 1989 ; revision accepted for publication September 30, 1989.

Address for reprint requests : Toru Sakuma, M.D., Department of Pediatrics, Nagoya City University Medical School, 1 Kawasumi, Mizuho-ku 467, Nagoya, Japan. 
was the kind gift of Earth Chemical Co. (Tokyo). Benzoylchloride was purchased from Wako Pure Chemical Industries (Tokyo). Authentic benzoylcarnitine was synthesized by Bohmer and Bremer's alternate method (Bohmer and Bremer 1968). Its final purity was over $95 \%$.

\section{Methods}

Fast atom bombardment mass spectrometry (FAB/MS) spectrum of each sample was recorded on the JMS DX-300 double focussing mass spectrometer (JEOL, Tokyo) at low resolution, equipped with the manufacture's $\mathrm{FAB}$ ion source and sample introduction system. Two microliters of sample solution prepared for FAB/MS with $20 \mu \mathrm{l}$ of methanol/ 3.5 $\mathrm{N}$ hydrochloric acid was applied to a stainless steel target, on which glycerol as matrix was coated smoothly. Six $\mathrm{KeV}$ fast argon atom was used to bombard an ionize the sample (Millington et al. 1984), and constant B/E ratio linked scanning was also performed.

Liquid chromatograms were obtained by a carboxylic acid analyser (Model S-14 CAA ; Tokyo Rika Kikai, Tokyo) connected with a CLC-ODS reverse phase column $(6 \mathrm{~mm} \times 15 \mathrm{~cm}$, Shimadzu, Kyoto) (Kidouchi et al. 1987). The eluent used for the gradient system was a water-acetonitrile at the concentration from $3 \%$ to $25 \%$ during $40 \mathrm{~min}$ at $37^{\circ} \mathrm{C}$. The injection volume was $100 \mu \mathrm{l}$ of urine sample, which was prepared with a cation exchange

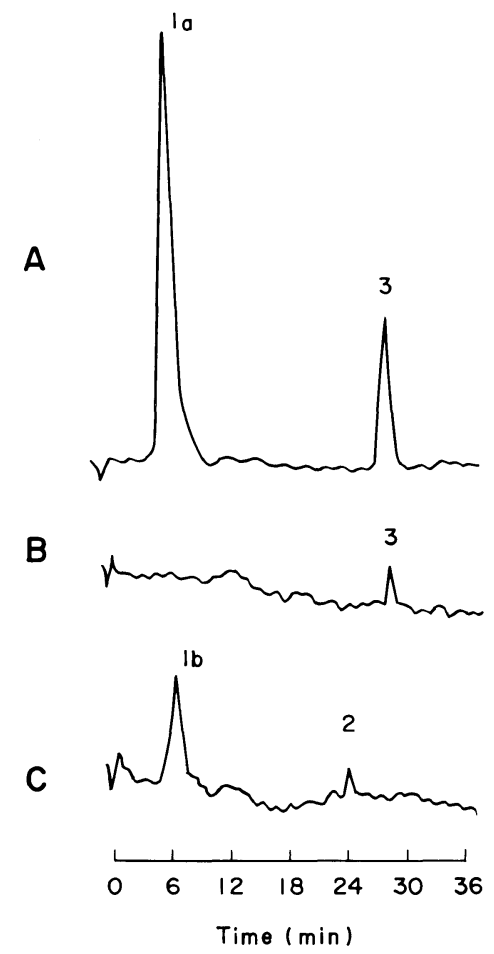

Fig. 1. Chromatograms obtained by a CAA with an ODS column. A : urine sample. B : fractionated urine sample. C: hydrolized sample of B. la, peak composed of carnitine, amino acids and some organic acids ; $1 \mathrm{~b}$, peak of carnitine; 2, peak of benzoic acid; 3, peak of benzoylcarnitine. Carnitine peak is 3.2 times as high as benzoylcarnitine peak at the same molarity. 
column, pyridine type (MCI GEL CKO8P ; Mitsubishi Chemical, Tokyo). In accordance with the retention time of authentic standard with a $\mathrm{CAA}$, the fraction including benzoylcarnitine was separated, and then lyophilized. The residue dissolved in $400 \mu 1$ of deionized water was divided equally into two parts. The one was analysed using a CAA fitted with an ODS column, and the other was cone similarly after hydrolysis.

\section{RESUlTS}

The chromatogram of the patient's urine revealed two large peaks. The first was a complexed figure consisted of free carnitine, amino acids and some organic acids, and the second was that of benzoyl compound. After hydrolysis, the latter peak (Fig. 1, peak 3) disappeared but instead of that, two new peaks appeared on the chart. They proved to be carnitine (Fig. 1, peak 1b) and benzoic acid (Fig. 1 , peak 2), respectively. Therefore we compared the second peak without hydrolysis, to that of benzoylcarnitine. The spectra of the methyl esters of authentic deuterium labeled and urinary unlabeled benzoylcarnitine with the constant B/ E ratio linked scanning are shown in Fig. 2. Both benzoylcarnitine fragmentation profiles coresponded, only except for the difference of deuterium exchange.

Both calibration curves for free L-carnitine and benzoylcarnitine were linear

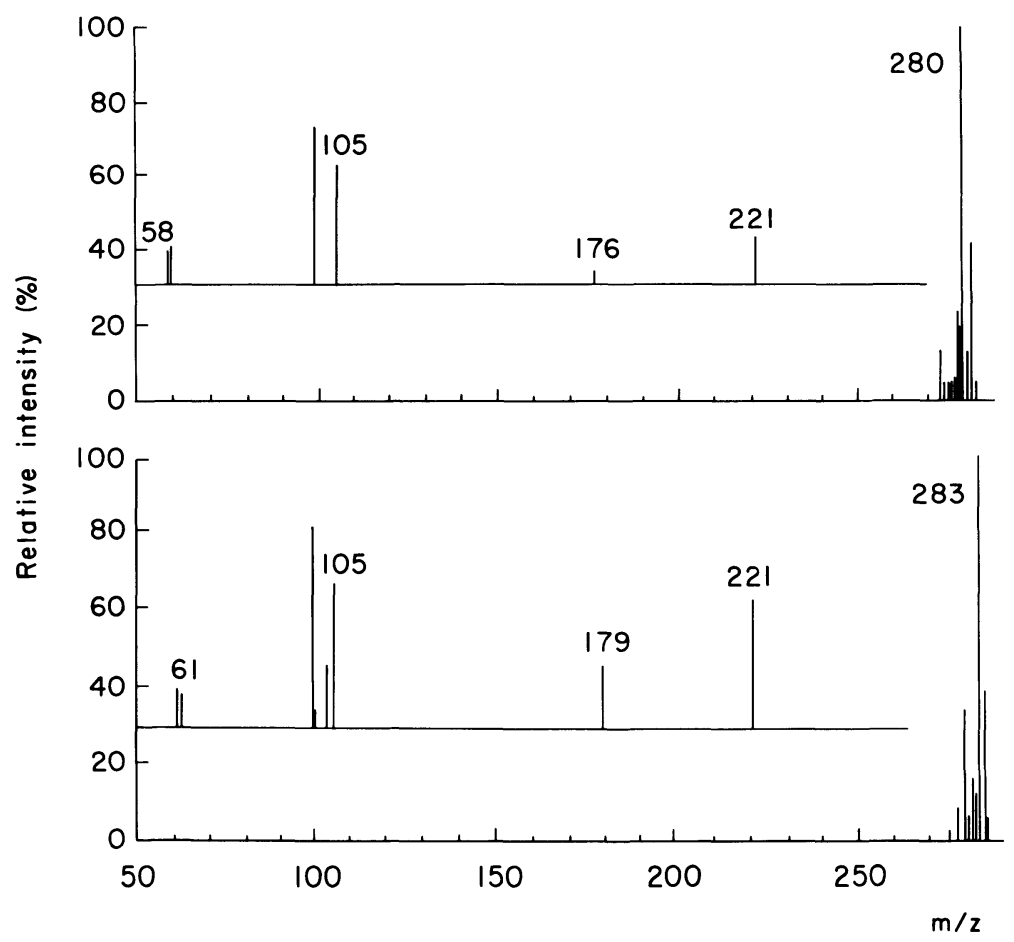

Fig. 2. Constant $\mathrm{B} / \mathrm{E}$ ratio linked scanning spectra of unlabeled benzoylcarnitine methyl ester (upper ; m/z 280) and authentic labeled benzoylcarnitine (lower ; $\mathrm{m} / \mathrm{z} 283$ ). 


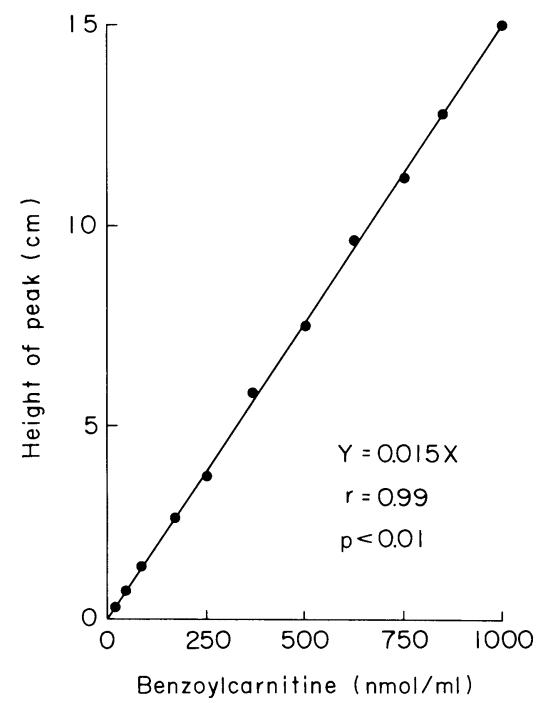

Fig. 3. Calibration curve of benzoylcarnitine by a CAA with an ODS column. $\mathrm{r}=0.99, p<0.01$.

over the sample concentration range from 10 to $1000 \mathrm{nmol} / \mathrm{ml}$ (Fig. 3), but only benzoic acid was quantified under $500 \mathrm{nmol} / \mathrm{ml}$ by this elution system. The carnitine peak was 3.2 times as high as the benzoylcarnitine peak at the same molarity. The range of recovery (mean \pm S.D., $n=5$ ) of the benzoylcarnitine in urine was $58.4 \pm 6 \%$, and its concentrations in urine were from 0.10 to $2.25 \mathrm{mmol} / \mathrm{g}$ creatinine in this preliminary examination.

\section{Discussion}

It is well-known that long-term therapy with protein restriction and sodium benzoate results in remarkable clinical improvement in patients with hyperammonemia, but the fate of benzoate, except for the portion used for the biosynthesis of hippurate is not clear. Recently, it was reported that, in experimental animals, sodium benzoate potentiated ammonia toxicity and inhibited urea systhesis in vivo, and benzoate increased mortality and levels of blood amnonia (O'Connor et al. 1987). Further, the presence of secondary carnitine deficiency overlapped to primary hyperammonemia, has been recognized in patients treated with sodium benzoate. L-Carnitine administration for these cases is effective to reduce benzoate toxicity and the frequency of hyperammonemic attacks (Matsuda et al. 1987). Since carnitine plays an important role in the regeneration of intramitochondrial free CoASH from excess acyl-CoA, benzoylcarnitine biosynthesis would serve to maintain cellular stability because one of the intermediates of benzoate is benzoyl-CoA.

Benzoylcarnitine was identified for the first time in this report. Further investigation must be made of the physiological significance of benzoylcarnitine. 


\section{References}

1) Bohmer, T. \& Bremer, J. (1968) Propionylcarnitine, physiological variations in vivo. Biochim. Biophys. Acta, 152, 297-307.

2) Kidouchi, K., Sugiyama, N., Morishita, H. \& Wada, Y. (1987) Identification of glutarylcarnitine in glutaric aciduria type 1 by carboxylic acid analyser with an ODS reverse-phase column. Clin. Chim. Acta, 164, 261-266.

3) Matsuda, I., Ohtani, K., Ohyanagi, K. \& Yamamoto, S. (1987) Hyperammonemia related to carnitine metabolism with particular emphasis on ornithine transcarbamylase deficiency. Enzyme, 38, 251-255.

4) Millington, D.S., Roe, C.R. \& Maltby, D.A. (1984) Application of high resolution fast atom bombardment and constant $\mathrm{B} / \mathrm{E}$ ratio linked scanning to the identification and analysis of acylcarnitine in metabolic disease. Biomed. Mass Spectrom., 11, 236241.

5) O'Connor, J.E., Costell, M. \& Grisolia, S. (1987) The potentiation of ammonia toxicity by sodium benzoate is prevented by L-carnitine. Biochim. Biophys. Res. Commun., 145, 817-824. 